\title{
Quenching of singlet oxygen by natural and synthetic antioxidants and assessment of electronic UV/Visible absorption spectra for alleviating or enhancing the efficacy of photodynamic therapy
}

\author{
Kaneez Fatima, Nusrat Masood, Suaib Luqman*
}

Molecular Bioprospection Department of Biotechnology Division, CSIR-Central Institute of Medicinal and Aromatic Plants, Lucknow226015, Uttar Pradesh, India

*Corresponding author: s.luqman@cimap.res.in

Received: 11 Feb 2016 / Accepted: 23 Feb 2016 / Published online: 30 Feb 2016

(C) The Author(s) 2016. This article is published with open access by BioMedPress (BMP)

\begin{abstract}
Photodynamic therapy (PDT) is one of the methods involved in cancer treatment exploiting singlet oxygen as a weapon to kill cancer cells. Singlet oxygen, a bizarre reactive oxygen species as it is not related to electron transfer to $\mathrm{O}_{2}$ but it is one of the most active intermediate involved in biochemical reactions as it directly reacts with all the major macromolecules like DNA, protein, lipids etc, various photosensitized oxidations and in the photodegradation of dyes and polymers. Recent studies about the usage of antioxidant along with the photo-sensitizer involved in photodynamic therapy have shown concentration- dependent dual behavior like usually it retards the efficacy of PDT but at a higher dose , it actually enhances the damaging effect of PDT. The natural and synthetic antioxidants are being used in our day to day life in order to increase the shelf life of various food ingredients and processed foods. In this paper, we have compared natural and synthetic antioxidants along with the known singlet oxygen quencher (DABCO) in order to understand the quenching potential of singlet oxygen $\left({ }^{1} \mathrm{O}_{2}\right)$ which is lowest electronically excited state of molecular oxygen. The singlet oxygen can be artificially generated through various methods such as sunlight, phosphate, ozonides, $\mathrm{NaOCl} \& \mathrm{H}_{2} \mathrm{O}_{2}$ etc. We have studied the mechanisms of selected antioxidants on the bleaching of RNO linked with the energy decay of ${ }^{1} \mathrm{O}_{2}$ produced by the Mallet reaction $\left(\mathrm{H}_{2} \mathrm{O}_{2}+\right.$ $\mathrm{HOCl} \rightarrow \mathrm{HCl}+\mathrm{H}_{2} \mathrm{O}+{ }^{1} \mathrm{O}_{2}$ ). $\beta$-Carotene, $\alpha$-Tocopherol, Ascorbic acid and Quercetin exhibited best dosedependent singlet quenching ranging from 92.3 to $56.5 \%$ at $100 \mu \mathrm{M}$ among others. Overall singlet oxygen is a major concern of light-related properties so we have analyzed the theoretical aspect of electronic UV/visible absorption spectra of the antioxidants studied through ZINDO CI semi-empirical Hamiltonian method. We have compared only the first singlet state of molecules in order to understand whether the structure of the molecules has any influence on the quenching potential. Considering the main features associated with singlet states, we have seen a drastic correlation between known sensitizer of singlet oxygen, i.e. Rose Bengal and DABCO, known scavenger of singlet oxygen in terms of energy and wavelength. This study is important as it highlights the behavior of antioxidant which can improve the effects of photosensitizer used in photodynamic therapy.
\end{abstract}

Keywords - Singlet oxygen; ${ }^{1} \mathrm{O}_{2}$; Photodynamic therapy; Antioxidant; RNO; Singlet state; ZINDO CI

\section{INTRODUCTION}

In 1931, singlet oxygen was predicted by Kautsky as a likely reaction intermediate in dye-sensitized photooxygenations (Ameta, 1990), but in 1964 it was fully established that singlet oxygen was same whether generated in sensitized photo-oxygenations, radio frequency generated or chemically generated using $\mathrm{H}_{2} \mathrm{O}_{2} / \mathrm{NaOCl}$ (Greer, 2006). Chemically generated singlet oxygen was obtained through acidification of hypochlorite in aqueous solution at around $\mathrm{pH}$ 7.5. This system of production of singlet oxygen has dual profit as it occurs at physiologically biological $\mathrm{pH}$ as 


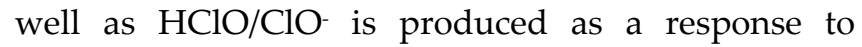
inflammation, therefore, this reaction may represent an inherent source of singlet oxygen in biological systems (Jacobsen and Jensen, 2007). Thus, the combination of equal molarity of hypochlorous acid and hydrogen peroxide produce singlet oxygen. $\mathrm{H}_{2} \mathrm{O}_{2}$ $+\mathrm{NaOCl} \rightarrow \mathrm{O}_{2}\left({ }^{1} \Delta_{\mathrm{g}}\right)+\mathrm{NaCl}+\mathrm{H}_{2} \mathrm{O}$ (Jacobsen and Jensen, 2007).

Antioxidants, as the name suggests are assumed as well as reported to be protective against damaging effect due to photo-oxidation (Jakus and Farkas, 2005; Stockert and Herkovits, 2003) yet sometimes the behavior may change for example in the presence of catalytic metals or at higher concentration they exhibit pro-oxidant activity (Jakus and Farkas, 2005; Shevchuk et al., 1998). In the case of PDT, some antioxidant molecules are reported to enhance the photodamaging effect of photosensitizer used in photodynamic therapy with various sensitizers and tumor models such as $\alpha$-Tocopherol (Jakus and Farkas, 2005; Melnikova et al., 1999), Ascorbic acid (Bachowski et al., 1988; Buettner et al., 1993; Girotti et al., 1985; Jakus and Farkas, 2005; Kaliya et al., 1999; Kelley et al., 1997; Rosenthal and Ben-Hur, 1992), water-soluble vitamin E analog, Trolox (Jakus and Farkas, 2005; Melnikova et al., 2000) and BHA (Jakus and Farkas, 2005; Shevchuk et al., 1998). Therefore, few antioxidants can exaggerate the effects of photodynamic therapy with unknown mechanism. Probably, the presence of higher antioxidant concentrations in cells, rationalizes the mechanism of PDT action from ${ }^{1} \mathrm{O}_{2}$-mediated route towards a primarily free-radical-mediated one as evidenced by the work of Bachowski and coworkers (Bachowski et al., 1991; Jakus and Farkas, 2005) showing that in the presence $1 \mathrm{mM}$ ascorbic acid and iron, irradiation of the photo-sensitizer MC540 produced drastically less primary ${ }^{1} \mathrm{O}_{2}$-adducts and produced various free radical mediated products in human erythrocyte ghosts (Jakus and Farkas, 2005).

Any photosensitizing compound absorbs radiant energy may raise its electronic state from the ground level by electronic rearrangement to an excited singlet state. The further transformation may lead to the triplet state, which when reacts with molecular oxygen; generate singlet oxygen. Similarly, molecules which are good quenchers also exhibit some structural properties which help in efficient quenching of singlet oxygen molecules. There are a lot of work on properties and reactions on different chemical and biological properties but no report is present on the comparative electronic state properties of the molecules in their singlet states which possess properties like sensitizer or quencher of singlet oxygen. Singlet oxygen has low LUMO energy and thus behaving as an electrophile which can react easily with electron rich double bonds (http://www.chem.wisc.edu/areas/reich/chem547/2redox\%7B26\%7D.htm). The quenching of singlet oxygen occurs due to the deactivation of the excited state of molecule which is either physical (no oxygen consumption or product formation for example sodium azide and DABCO) or chemical type of quenching (for example quenching by carotene, ascorbate, thiols and histidine) which is further of two types, the first by reversing the reaction of singlet oxygen production known as energy transfer quenching and secondly through the interaction between the electron-deficient ${ }^{1} \mathrm{O}_{2}$ molecule and electron donors to form a charge transfer complex known as Charge transfer quenching. Singlet oxygen is quenched by or reacts with many organic and bioorganic molecules, which possess, in most cases, reactive $\pi$ electrons or $n$ lone pairs of sufficiently low ionizing energy. Singlet oxygen quenching or scavenging may be via physical method (the quencher enters a vibrational or an electronic excited state) or through chemical method (the quencher combines with oxygen or is oxidized by oxygen) in nature. Physical quenching may happen by catalysis of the singlet oxygen $\left({ }^{1} \mathrm{O}_{2}\right)-->$ ground state oxygen $\left({ }^{3} \mathrm{O}_{2}\right)$ transition via a spin-orbit coupling or via triplet energy transfer. Charge transfer reactions are important during the quenching process. Well established singlet oxygen quenchers such as azide, 1,4 diazabicyclo [2.2.2] octane (DABCO) or $\beta$-carotene (Wilkinson et al., 1995). A spectrophotometric method of detection of singlet oxygen reaction through secondary reaction in which yellow colored $p$-nitroso dimethylaniline (RNO) is used as an indicator dye through the help of singlet oxygen trapper (imidazole or its derivative, L-Histidine) was developed by Kraljic and Mohsni (1978). They further interpreted that as RNO does not chemically react with singlet oxygen $\left({ }^{1} \mathrm{O}_{2}\right)$, bleaching was due to the formation of a transannular peroxide intermediate after ${ }^{1} \mathrm{O}_{2}$ capture by the imidazole ring. By combining chemically generated production of singlet oxygen (through $\mathrm{NaOCl} \& \mathrm{H}_{2} \mathrm{O}_{2}$ ) and $p$-nitroso dimethylaniline (RNO)Histidine based bleaching by singlet oxygen, we analyzed the comparative evaluation of some natural 
and synthetic antioxidants which are present in our body or used in day to day life as food preservatives. Also at the same time, we also want to assess the biophysical properties of these molecules, especially in their first excited state. In our body generation of singlet oxygen is obligatory because it is an active intermediate molecule, involve in various biochemical reactions. But due to any physiological stress or in other conditions, there is a significant increase in singlet oxygen $\left({ }^{1} \mathrm{O}_{2}\right)$ as a result; it can be toxic to biomolecules and thus cause several diseases including cancer. Singlet oxygen is reported to be cytotoxic or genotoxic to mammalian cell culture (Thomas et al., 2002). Biological targets for singlet oxygen comprise DNA, unsaturated fatty acids, protein, lipids etc. On several instances, singlet oxygen is responsible for induction of tumor by photosensitization, metabolic activation of carcinogen and highly deleterious the membrane of lipid or protein moieties. In mammalian biology, ${ }^{1} \mathrm{O}_{2}$ causes oxidation of LDL cholesterol as a consequence of cardio vasculature damage, accountable for several pathological process including lung oxidant injury, erythropoietic porphyria, skin photosensitivity and some report show that singlet oxygen incriminates in inactivation of a cell or cellular component due to ultraviolet and visible rays (Koichi et al., 2011). The occurrence of singlet oxygen is not only limited to cells but also find in the atmosphere due to pollution (e.g. polyaromatic hydrocarbons) as well as found in aquatic systems (Hooper et al., 2013). Molecular oxygen exhibits a remarkable electronic structure, as its higher occupied electronic level is constituted of two $\pi^{*}$ orbitals of the same energy, socalled degenerated, filled with only two electrons (DeRosa \& Crutchley, 2002). In the ground state, each of these electrons lies in one $\pi^{*}$ orbital and their spins are parallel, hence, it is a triplet state denoted by ${ }^{3} \mathrm{O}_{2}$ $\left({ }^{3} \sum \mathrm{g}^{-}\right)$. It behaves chemically as a weakly reactive oxidizing diradical despite its elevated oxidation potential (DeRosa \& Crutchley, 2002). The first excited state known as singlet state of oxygen or singlet oxygen denoted by ${ }^{1} \mathrm{O}_{2}$ or $\left({ }^{1} \Delta_{\mathrm{g}}\right)$ has both electrons in the same orbital with opposite spins, and exhibits longer lifetime (45 $\mathrm{min}$ in vacuum and $4 \mu \mathrm{sec}$ in water) and a substantial reactivity towards electron-rich organic molecules such as dienes, olefins, phenols and polycyclic aromatic compounds. Octanol/water partition $(\log \mathrm{P})$ coefficient are generally used to estimates for membrane penetration and permeability, blood brain barrier and gastrointestinal absorption (Pajouhesh \& Lenz (2005).
We have studied the mechanisms of selected antioxidants on the bleaching of RNO linked with the energy decay of ${ }^{1} \mathrm{O}_{2}$ produced by the Mallet reaction $\left(\mathrm{H}_{2} \mathrm{O}_{2}+\mathrm{HOCl} \rightarrow \mathrm{HCl}+\mathrm{H}_{2} \mathrm{O}+{ }^{1} \mathrm{O}_{2}\right)$. Overall, singlet oxygen is a major concern of light-related properties so we also analyzed the electronic UV/visible absorption spectra. The method used is the ZINDO semi-empirical Hamiltonian of Zerner and coworkers. First a ground-state closed shell SCF calculation was done and then configuration interaction [CI] calculation was performed using single-excited configurations in order to solve for the excited states (Osman et al., 2014).

\section{MATERIALS AND METHODS}

\section{Chemicals}

N,N-dimethyl-4-nitrosoaniline (RNO), NaOCl, Lhistidine, L-Ascorbic acid, Trolox, 1,4-diazabicyclo [2.2.2] octane (DABCO) and dimethyl sulfoxide (DMSO) were purchased from Sigma-Aldrich, USA while butylated hydroxyanisole (BHA), butylated hydroxytoluene (BHT), tertbutyl hydroquinone (TBHQ), tocopherol, quercetin, nicotinic acid and $\beta$ carotene were purchased from Himedia, India. Disodium hydrogen phosphate $\left(\mathrm{Na}_{2} \mathrm{HPO}_{4}\right)$, sodium dihydrogen phosphate $\left(\mathrm{NaH}_{2} \mathrm{PO}_{4}\right), \mathrm{H}_{2} \mathrm{O}_{2}$, ethanol, methanol and acetone was purchased from Merck India Ltd. All the chemicals, solvents and reagents used were of analytical grade or higher.

\section{Experimental}

Stock solution of $0.1 \mathrm{M}$ sodium phosphate buffer $(\mathrm{pH}$ 7.0) was made by mixing solutions of $\mathrm{Na}_{2} \mathrm{HPO}_{4}(0.1$ M) and $\mathrm{NaH}_{2} \mathrm{PO}_{4}(0.1 \mathrm{M})$. Stock solutions of $1 \mathrm{mM}$ $\mathrm{N}, \mathrm{N}$-dimethyl-4-nitrosoaniline (RNO); $0.7 \mathrm{M} \mathrm{NaOCl}$; $1 \mathrm{M} \mathrm{H}_{2} \mathrm{O}_{2} ; 100 \mathrm{mM}$ L-histidine; 100mM DABCO; $10 \mathrm{mM}$ stock solution of BHT, L-ascorbic acid, Trolox, nicotinic acid, and hydrogen peroxide was made in deionized water, Tocopherol and BHA was made in ethanol, TBHQ in methanol, quercetin in DMSO, and $\beta$ carotene in acetone. The concentrations used for the analysis were $100 \mu \mathrm{M}, 10 \mu \mathrm{M}, 1 \mu \mathrm{M}$, and $0.1 \mu \mathrm{M}$.

\section{Singlet oxygen trapping assay}

The production of singlet oxygen $\left({ }^{1} \mathrm{O}_{2}\right)$ was determined by monitoring $\mathrm{N}, \mathrm{N}$-dimethyl-4nitrosoaniline (RNO) bleaching (Kraljic \& Mohsni, 
1978). In final volume of $200 \mu \mathrm{l}$, final working concentration of sodium phosphate buffer ( $\mathrm{pH}$ 7.0) was $25 \mathrm{mM}$, RNO $(100 \mu \mathrm{M}), \mathrm{NaOCl}(100 \mathrm{mM}), \mathrm{H}_{2} \mathrm{O}_{2}$ $(100 \mathrm{mM})$, L-Histidine $(12.5 \mathrm{mM})$. The system which chemically generates singlet oxygen has $\mathrm{NaOCl}$ and $\mathrm{H}_{2} \mathrm{O}_{2}$ and it was mixed in an overall reaction cocktail in equimolar ratio. After $10 \mathrm{~min}$ incubation, the absorbance was measured spectrophotometrically at $440 \mathrm{~nm}$ at $37^{\circ} \mathrm{C}$. The trapping capacity of sample was compared with control values and relative scavenging efficiency (\% inhibition in production of ${ }^{1} \mathrm{O}_{2}$ ) was estimated from the difference in absorbance with or without the addition of sample. Positive control is Rose Bengal and negative control/inhibitor DABCO. All tests were performed three times in duplicate.

\section{Quantum Chemical Studies for evaluating properties of molecules in their singlet states}

The 3D structure of all the molecules was taken from PubChem in the sdf format and converted to $\mathrm{pdb}$ format through OpenBabel 2.3.1 software. The structures were optimized by then an electronic excited state calculation (ie. absorption spectroscopy) was set up using the ZINDO semi-empirical Hamiltonian method of Zerner and co-workers by ArgusLab 4.0.1 software. The calculation consists of a ground-state closed shell SCF calculation followed by a configuration interaction calculation, using singleexcited configurations, to solve for the excited states. Also to understand the effect of solvent on these singlet states, the self-consistent reaction field (SCRF) solvent model of Zerner and co-workers was used. This helps in modeling the electronic spectra of a molecule in any given solvent. This is a continuum dielectric model. The ZINDO stands for Zerner's Spectroscopic parameterization of the Intermediate Neglect of Differential Overlap INDO1/s Hamiltonian. (Thompson, 1997; Zerner, 1991; Zerner et al., 1980).

\section{Statistical Analysis}

Absorbance is taken through SoftMax Pro Microplate Data Acquisition and Analysis Software Version 5.3 (Molecular Devices Corporation, Sunnyvale CA, USA) Data represented are mean/average \pm standard deviation of three independent experiments in duplicate.

\section{RESULTS AND DISCUSSION}

The natural and synthetic as well as lipophilic and hydrophilic nature of antioxidants manifest varied behavior to singlet oxygen in a concentrationdependent manner $[0.1 \mu \mathrm{M}-100 \mu \mathrm{M}$ (Fig. 1)]. LHistidine or Imidazole has been used as a trapper of singlet oxygen in $p$-nitrosodimethylaniline based spectrophotometric detection of singlet oxygen (Fig. 2). L-Histidine have imidazole side chain and remain aromatic at all $\mathrm{pH}$ values. It has six $\pi$-electrons (due to double bond) and lone pair of nitrogen. It has tendency to move side chain from neutral to positive charge. Imidazole similarly possesses same properties. DAB$\mathrm{CO}$ was used as known scavenger of singlet oxygen (Fig. 3). Further the study on the known sensitizer, i.e. Rose bengal was observed through the system of bleaching of RNO by singlet oxygen (Fig. 4). The increase in production of singlet oxygen ranges from 23-37\% yet the effectiveness under the sunlight or UV light was more than through chemically generated singlet oxygen (Data not shown). Dose-dependent behavior is observed in this study regarding the quenching of singlet oxygen in which $\alpha$-Tocopherol exhibited best quenching potential with $\mathrm{IC}_{50}$ value of $0.528 \pm 0.095$ while $\beta$-carotene have an $\mathrm{IC}_{50}$ value of $3.59 \pm 0.12$. Apart from them, quercetin quenched $59.7 \%$ singlet oxygen at $100 \mu \mathrm{M}$ while ascorbic acid quenched $56.53 \%$ singlet oxygen at $100 \mu \mathrm{M}$. $\beta-$ Carotene belong to the carotenoid family and it is biosynthesized by tail to tail linkage from c 20 geranylgeranyl diphosphate molecule. It is discriminated from other carotenes by bearing $\beta$-ring at both end of the molecule. $\beta$-Carotene is a precursor of vitamin $\mathrm{A}$, has long aliphatic unsaturated hydrocarbon chain which cleaves into two adjacent molecules of vitamin A, an excellent scavenger of singlet oxygen. $\alpha$-Tocopherol, commonly called as vitamin $\mathrm{E}$, among eight types is a naturally occurring chemical compound found in varieties of foods. It provides protection against formation of singlet oxygen in a dose-dependent manner probably due to presence of chromane ring. Likewise, $\beta$-carotene and $\alpha$-tocopherol, quercetin also significantly scavenges singlet oxygen in a concentration dependent manner. Quercetin is a naturally occurring flavonoid found in bark of numerous plants. The I.U.P.A.C name of this phytochemical is 2- $(3,4-$ dihydroxyphenyl) -3,5,7-trihydroxy-4H-1benzopyran-4-one, scavenge and stabilize the free radical by active center of B-ring. Ascorbic acid, famous as vitamin $C$ serves as both one-electron and two- 
electron reducing agent. Singlet oxygen, being lowLUMO electrophile readily reacts with the reducing agent like ascorbate. The obtained results revealed that the singlet oxygen quenching activity of natural and synthetic antioxidant specifically of $\alpha$-tocopherol, $\beta$-carotene, quercetin and ascorbic acid was comparable with the known scavenger, DABCO.

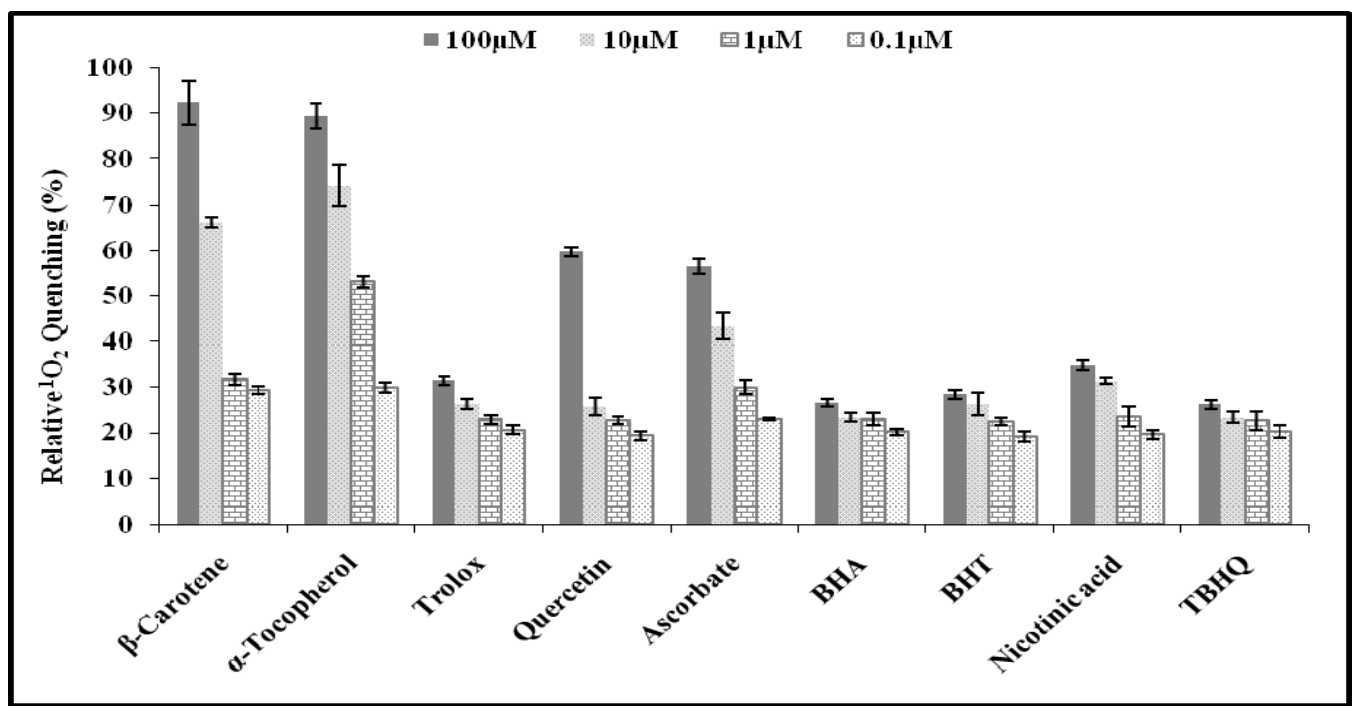

Figure 1. Relative scavenging potential of singlet oxygen by various natural and synthetic antioxidants.

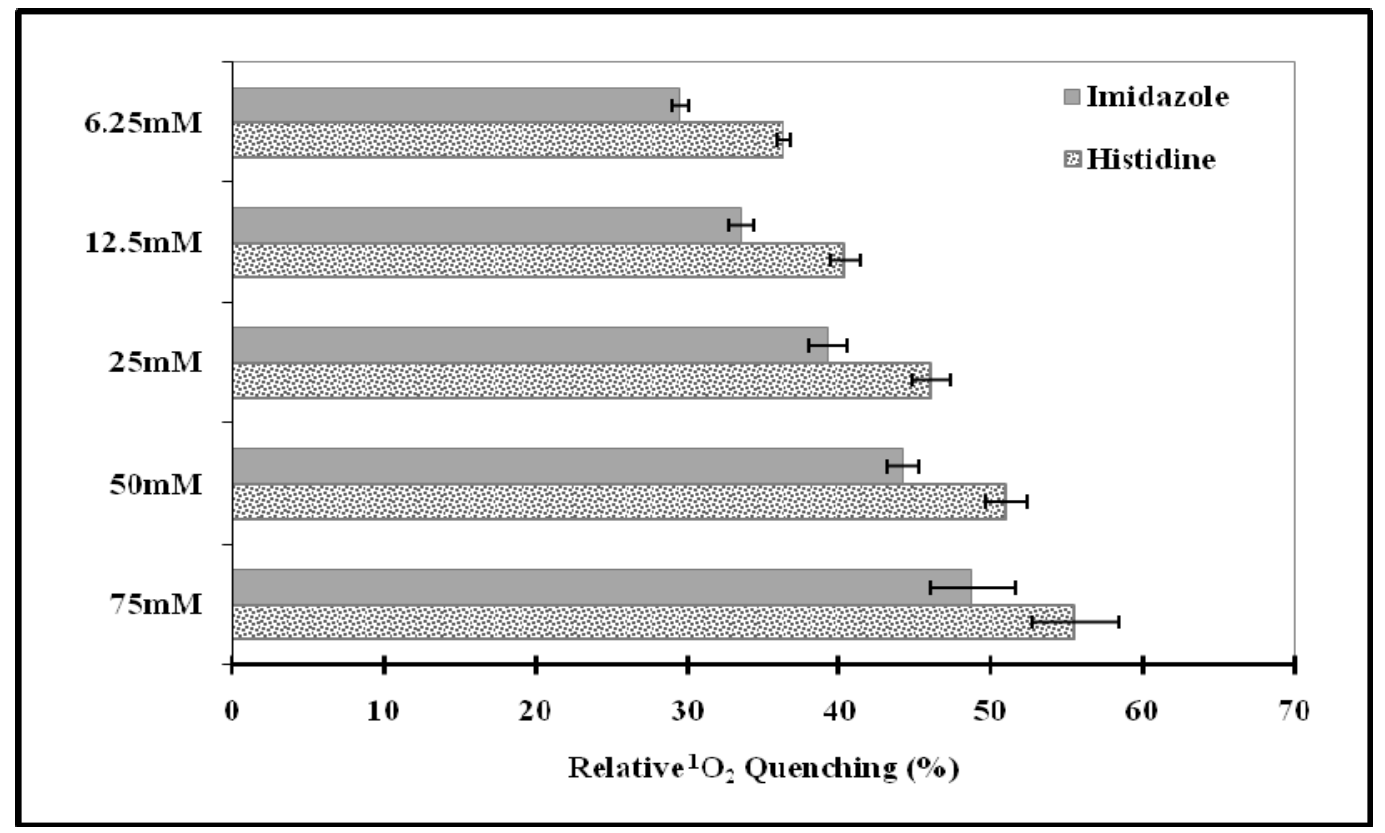

Figure 2. Relative scavenging potential of Histidine and Imidazole at different concentrations. 
Currently, only singlet excited states were calculated by ZINDO CI methods and this quantum-chemical calculation of electron-excited states was analyzed at the SCF level (an approximation of the ground state molecular orbitals and direct SCF calculation of excited states). Applications illustrated the suitability of the use of the methods (within semiempirical parametrizations) for interpretation of the properties of electron-excited organic molecules including photochemical reactions. The configuration interaction (CI) is the most general method for calculating the properties of the excited states. The present study revealed the comparative quantum absorption descriptors of different antioxidant as well as known scavenger and sensitizer towards singlet oxygen, in order to establish which molecule have a more drastic effect on all studied molecules. It is not a free radical but a strong oxidant since it has unique electronic configuration and $94.1 \mathrm{~kJ} / \mathrm{mol}$ higher energy than the ground state oxygen (Deby-Dupont et al., 1998). Singlet oxygen can cause damage in a wide range of biologically important molecules (Deby-Dupont et al., 1998). The involvement of singlet oxygen in a reaction is usually indirectly evaluated on the basis of the presence of characteristic products, such as "ene"-type products, or by using specific quenchers, such as 1,4diazabicyclo [2.2.2] octane (DABCO) or sodium azide.

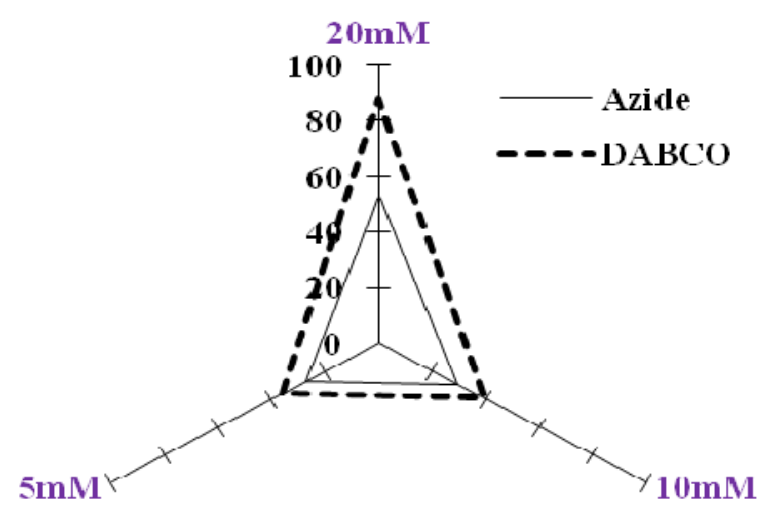

Figure 3. Scavenging potential of DABCO, a known quenchers of singlet oxygen.

For semiempirical calculation, the different structures were optimized and UV-Vis spectrum was calculated with ZINDO (Zerner's INDO) implemented in ArgusLab. Overall, 10 excited states were calculated of which various properties of only the first excited singlet state were plotted and compared. Further to mimic the experimental condition which occurs in the aqueous system, solvent contributions were incorporated using Self-Consistent Reaction Field approach (in SCRF the solvent is modeled as a continuous dielectric with the solute positioned at the center of a spherical cavity within the dielectric medium)

(http://www.usc.es/congresos/ecsoc/10/CC/g012/index $. \mathrm{htm})$. Electronic spectra of molecules were calculated via the method of ZINDO $\mathrm{Cl}$ in which SCRF solvent model was used in which water was used as a solvent with dielectric constant of 78.3, refractive index of 1.33 and mass density of $1 \mathrm{~g} / \mathrm{cc}$. The cavity radius was dis-

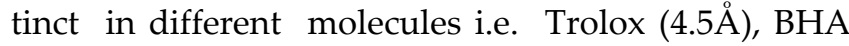
(4.66 ̊), BHT (5.05 $\AA)$, DABCO (4.02 $\AA$ ), Histidine $(4.34 \AA)$, Imidazole $(3.29 \AA)$, Nicotinic acid $(3.97 \AA), \beta$ Carotene (6.77 $\AA$ ), Quercetin (5.33 $\mathrm{A})$, RNO (4.31 $\AA$ ), Rose Bengal (6.02 $\AA$ ), TBHQ $(4.53 \AA)$ and $\alpha$ Tocopherol (6.34 ̊).

Fig. 5 and 6 were plotted comparing different molecules for their energy versus wavelength in their first excited state of vacuum and aqueous system respectively. Rose Bengal and DABCO exhibited drastic differences. Similarly, Fig. 7 and $\mathbf{8}$ were plotted comparing different molecules for their state dipole versus oscillator strength in their first excited state of vacuum and aqueous system respectively. $\beta$-Carotene and Quercetin exhibited drastic differences which may be due to their structural configuration. Fig. 9 and $\mathbf{1 0}$ compared the energy and wavelength at vacuum and aqueous system. In this only Rose Bengal was found to exhibit best solvatochromic shifts. These data were also tabulated and compared with the $\mathrm{H}$ bond donor, H-bond acceptor and $\mathrm{X} \log \mathrm{P} 3$ (https://pubchem.ncbi.nlm.nih.gov/) (Table 1-4). Rose Bengal have the lowest energy and highest wavelength while DABCO have the highest energy and lowest wavelength in both the systems (Table 1). Rose Bengal has the highest state dipole while DABCO have the lowest state dipole in both the systems (Table 2). Oscillator strength and H-Bond acceptor values were highly comparable, $\beta$-Carotene has the highest oscillator strength while Quercetin, have the lowest oscillator strength in both the systems. Similarly, H-bond acceptor obtained from PubChem follows a similar trend with $\beta$-Carotene having lowest (0) while Quercetin having the highest (7) H-bond acceptor count (Table 3). Oscillator strength refers to the probability that a molecule interacting with light over a certain energy range is going to absorb that 
light

(http://photonicswiki.org/index.php?title=Transition Dipole Moment). Further the leads among the tested molecules were plotted for their wavelength at all the 10 excited states (Fig. 11) in which Rose Bengal and RNO exhibited higher wavelength or less energy required to move to their first excited state while DABCO was almost constant in all the excited states. All the structures studied are depicted in Fig. 12. With the help of computational methods, we could predict the properties of excited electronic states. The oscillator strength conveys the potency of light absorption by a molecule from the integration of the $\mathrm{UV} /$ visible electronic absorption spectrum. It is proportional to the square of the magnitude of the transition dipole moment, i.e the dipole moment of the molecule induced by its interaction with the electric field of the incident radiation. For the very strong transition, the oscillator strength approximately equals to 1 and increases with the extension of the $\pi$ electron conjugation. The energy change associated with a transition between states is described by Planck's law $\Delta \mathrm{E}=\mathrm{h} v$, where $v$ is the frequency of the light emitted or absorbed. Because $v-c / \lambda$, where $c$ is the speed of light and $\lambda$ is the wavelength, there is an inverse relationship between energy and wavelength. The radiation less deactivation of an excited state is known as internal conversion (Frank and Christensen, 2008). In case of $\beta$-Carotene, the calculated data from quantum computations suggested that excited singlet state has actually a twisted conformational structure, the yield of which increases as the $\pi$-electron conjugated chain length of the molecule increases (Liaane-Jensen and Lutnees, 2008). A singlet state has no net spin angular momentum because it is an electronic state of a molecule in which all the electronic spins are paired (Suppan, 1990). Nature and the energy of electronically excited state of a dye molecule determine its photo-physical properties. In quantum calculations, the molecule is assumed absolutely to be isolated, as if it were in the gas phase at very low pressure. Since we are concerned with molecules in solvents, especially in an aqueous system, the effect of electronic states of solute molecules is, therefore, of substantial importance in photodynamic therapy. The difference in the solvation energies of the final and initial states in various solvents is referred to as solvate-chromic shift and is the main experimental evidence for this energy difference (Ghazy et al., 2004). Therefore, the absorption and emission spectra are more informative in this respect as they are related to the energy of relaxed excited state. The Zerner's INDO method (ZINDO) is also called spectroscopic INDO (INDO/S). This is a re-parameterization of the INDO method specifically for the use of obtaining and predicting electronic spectra results. Thus, we have utilized ZINDO CI method for assessing our experimental results. Also, it may pave a way for evaluating more sensitizers and quenchers of singlet oxygen in the future.

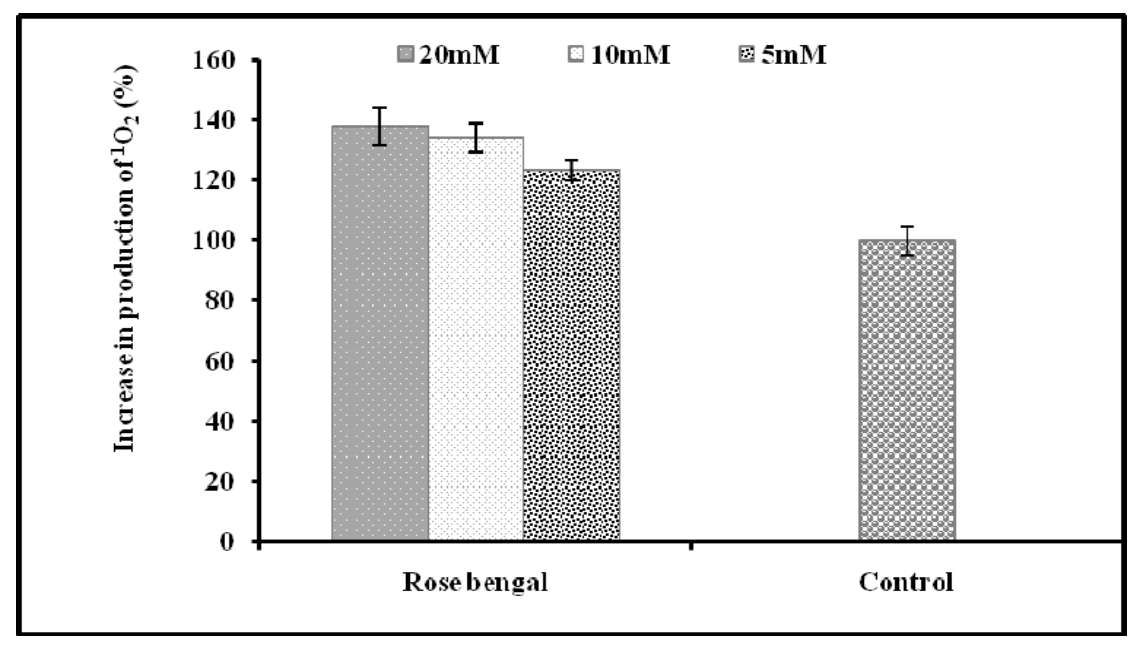

Figure 4. Percent activation in singlet oxygen production by Rose Bengal. 


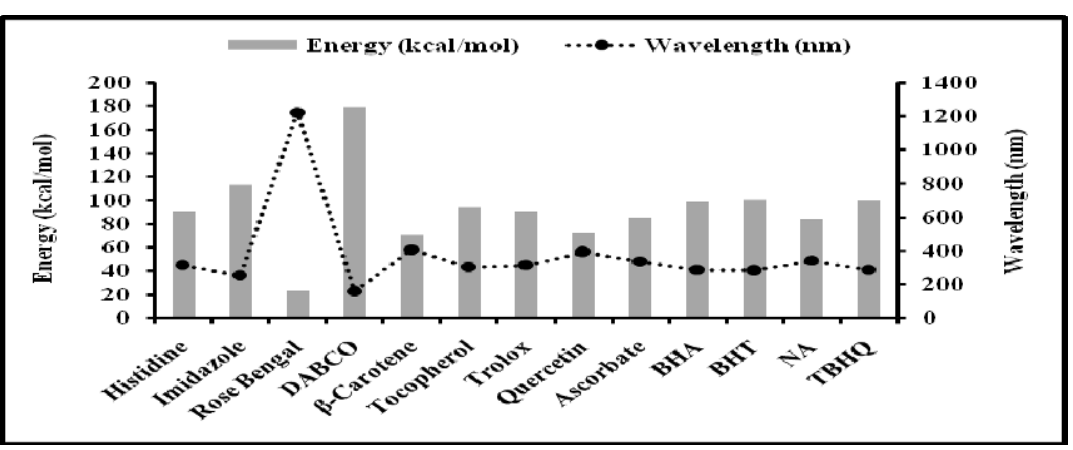

Figure 5. Comparative evaluation of molecules under study for their energy versus wavelength in their first singlet state of their excited electronic absorption spectrum. [Dielectric constant: 1 and Refractive index: 1(vacuum)]

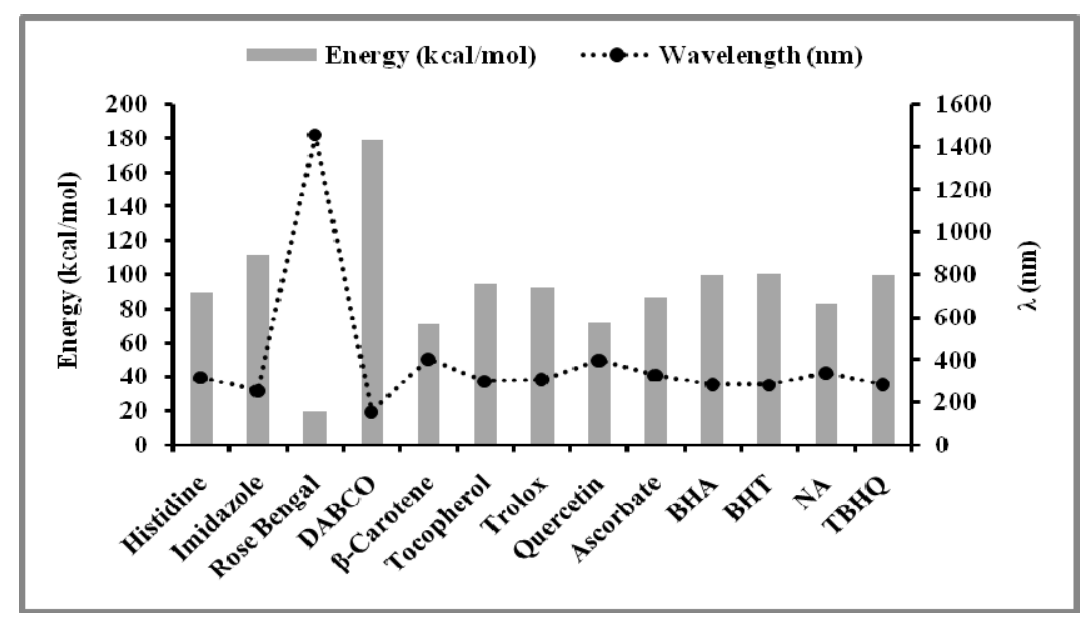

Figure 6. Comparative evaluation of molecules under study for their energy versus wavelength in their first singlet state of their excited electronic absorption spectrum. [Dielectric constant: 78.3 and Refractive index: 1.333 (water)]

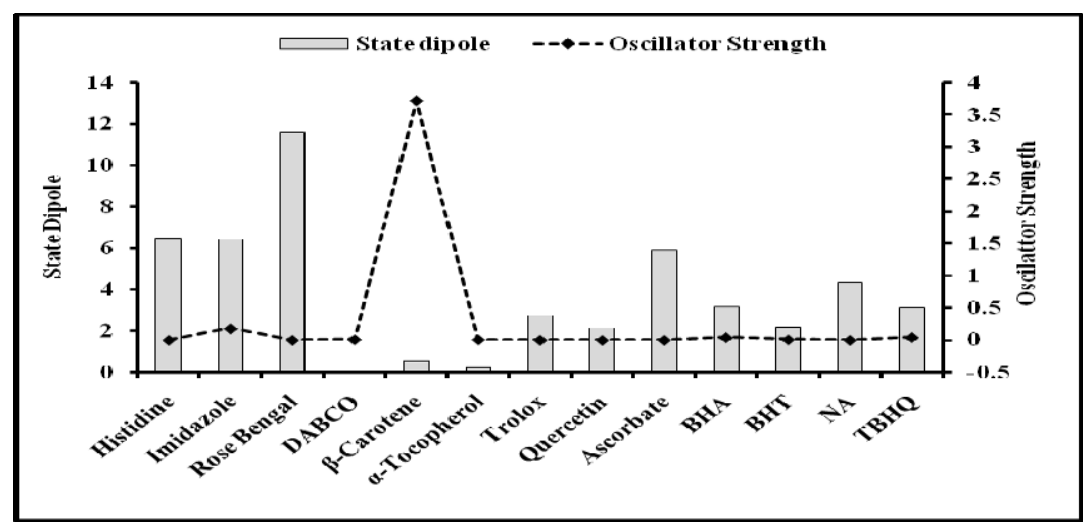

Figure 7. Comparative evaluation of molecules under study for their state dipole versus oscillator strength in their first singlet state of their excited electronic absorption spectrum. [Dielectric constant: 1 and Refractive index: 1 (vacuum)] 


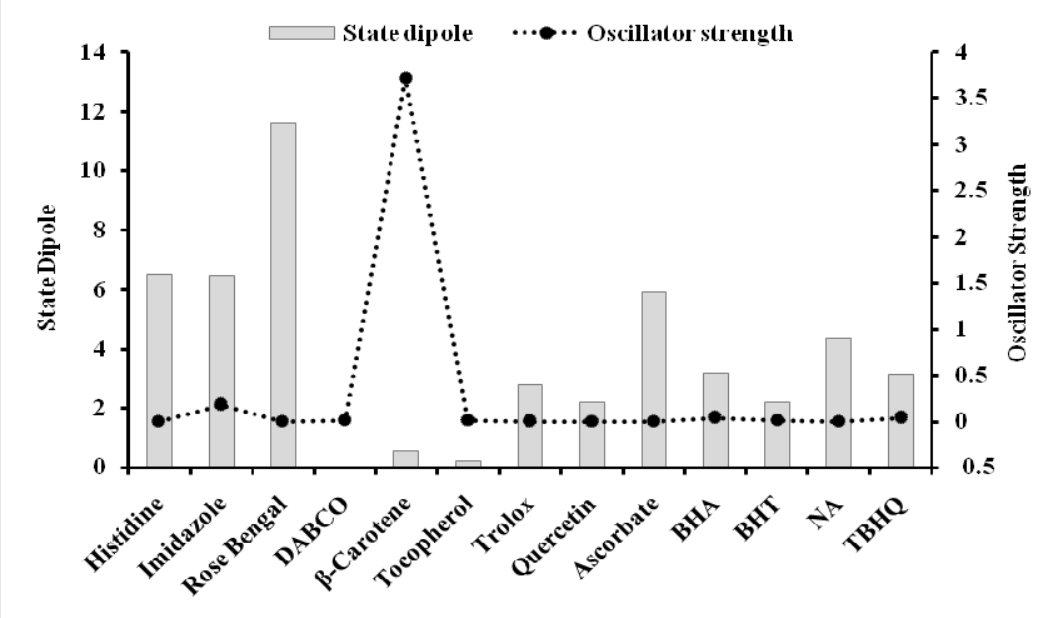

Figure 8. Comparative evaluation of molecules under study for their state dipole versus oscillator strength in their first singlet state of their excited electronic absorption spectrum. [Dielectric constant: 78.3 and Refractive index: 1.333 (water)]

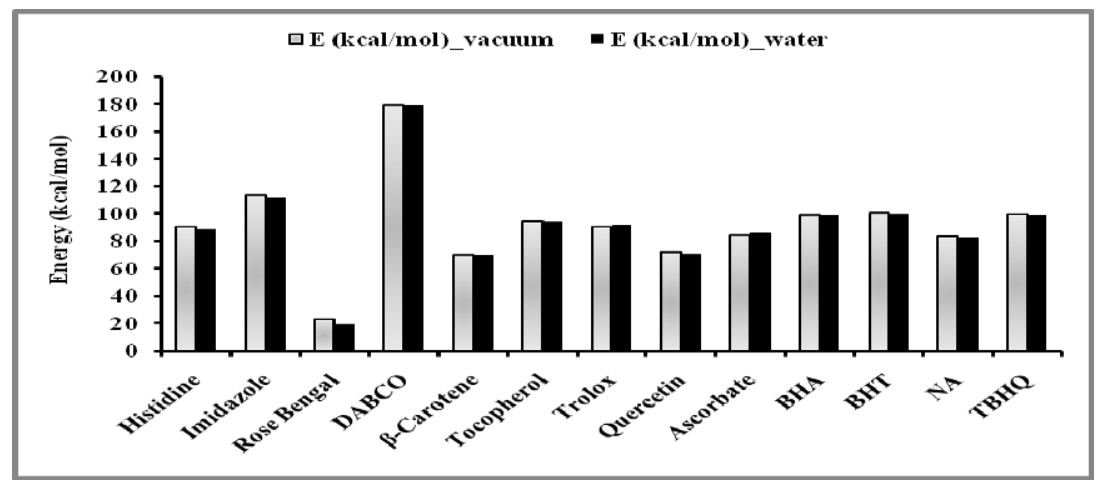

Figure 9. Comparative evaluation of molecules under study for their energy in both the systems (vacuum versus aqueous system) in their first singlet state of their excited electronic absorption spectrum.

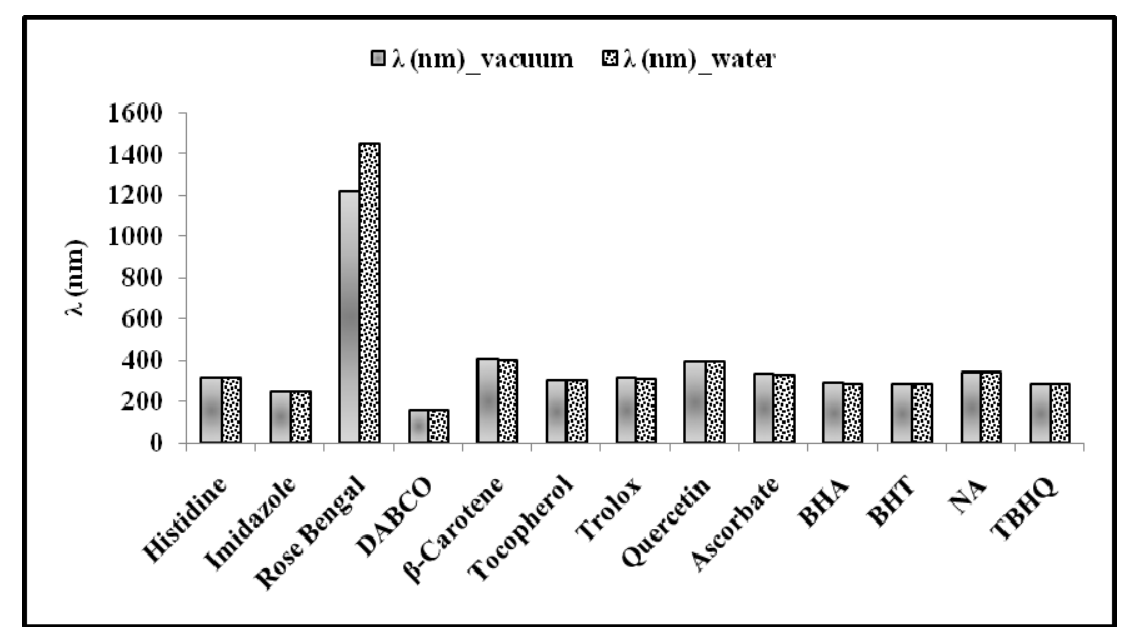

Figure 10. Comparative evaluation of molecules under study for their wavelength in both the systems (vacuum versus aqueous system) in their first singlet state of their excited electronic absorption spectrum.

Enhancing the efficacy of photodynamic therapy 


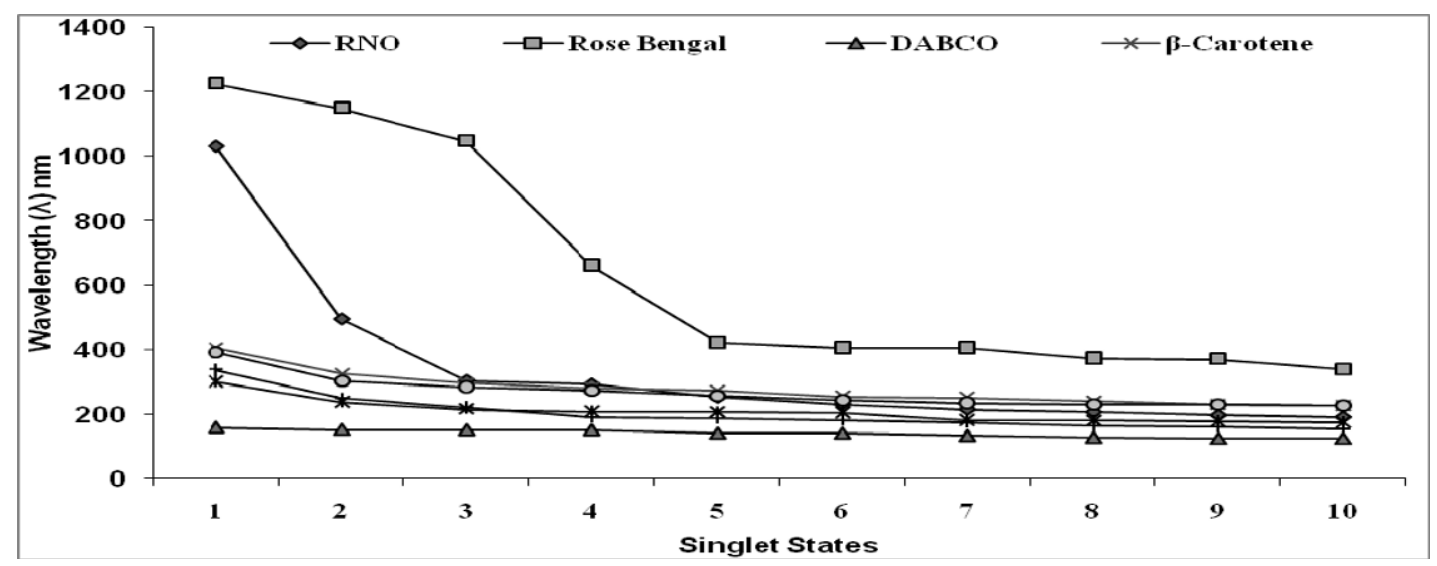

Figure 11. Comparative evaluation of main molecules under study for their wavelength in the aqueous system for all 10 singlet states of their excited electronic absorption spectrum.

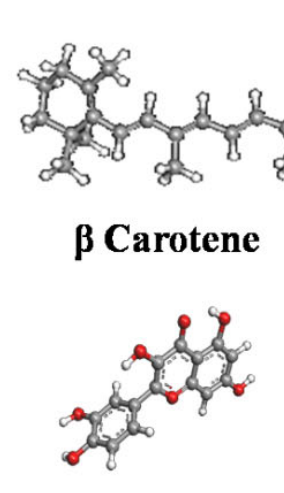

Quercetin

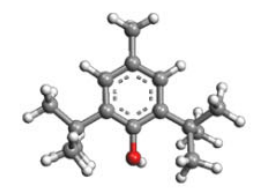

BHT

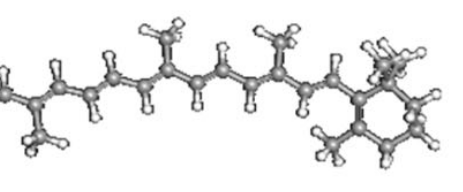

$\alpha$-Tocopherol

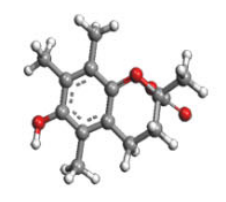

Trolox

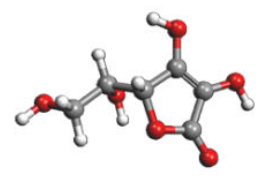

Ascorbic acid

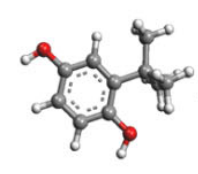

TBHQ
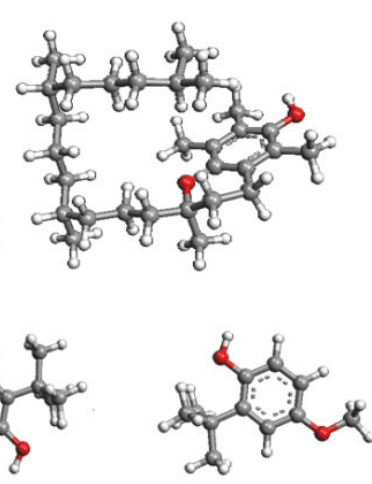

BHA

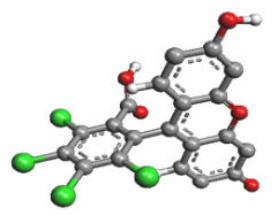

Rose Bengal

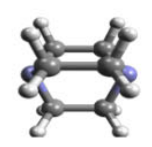

DABCO

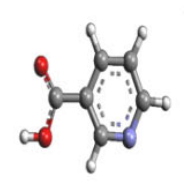

Nicotinic acid

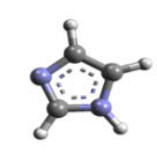

Imidazole

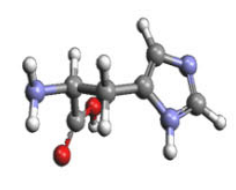

L-histidine

Figure 12. 3-Dimensional structure of all the molecules under study plotted using ArgusLab 4.0.1 viewer. 
Table 1. Comparative evaluation of molecules under study for their energy and wavelength in both the systems (vacuum versus aqueous system) in their first singlet state of their excited electronic absorption spectrum

\begin{tabular}{|l|l|l|l|l|}
\hline & \multicolumn{2}{|l|}{ Energy ${\left(\mathbf{c m}^{-1}\right)^{*}}^{*}$} & \multicolumn{2}{l|}{ Wavelength(nm) } \\
\hline & Vacuum & Water & Vacuum & Water \\
\hline Rose Bengal & 8159.2 & 6872.2 & 1225.4 & 1455.1 \\
\hline$\beta$-Carotene & 24669.1 & 24667.7 & 405.4 & 405.4 \\
\hline Quercetin & 25391.5 & 25011.4 & 393.8 & 399.8 \\
\hline Nicotinic acid & 29340.7 & 29166.7 & 340.8 & 342.9 \\
\hline Ascorbic acid & 29585.9 & 30261.8 & 338 & 330.4 \\
\hline L-histidine & 31676.1 & 31280.6 & 315.7 & 319.7 \\
\hline Trolox & 31716.9 & 32218.4 & 315.3 & 310.4 \\
\hline$\alpha$-Tocopherol & 33133.3 & 33133.5 & 301.8 & 301.8 \\
\hline BHA & 34814.5 & 34801.5 & 287.2 & 287.3 \\
\hline TBHQ & 34955 & 34934.2 & 286.1 & 286.3 \\
\hline BHT & 35207 & 35207.1 & 284 & 284 \\
\hline Imidazole & 39700.4 & 39252 & 251.9 & 254.8 \\
\hline DABCO & 62735.8 & 62735.8 & 159.4 & 159.4 \\
\hline
\end{tabular}

*Arranged from lowest to highest ** Arranged from highest to lowest

Table 2. Comparative evaluation of molecules under study for their state dipole in both the systems (vacuum versus aqueous system) in their first singlet state of their excited electronic absorption spectrum

\begin{tabular}{|l|l|l|l|}
\hline \multicolumn{2}{|l|}{ State dipole_vacuum } & \multicolumn{2}{l|}{ State dipole_water ${ }^{* *}$} \\
\hline Rose Bengal & 11.58975 & 18.10078 & Rose Bengal \\
\hline L-histidine & 6.473551 & 7.120597 & Imidazole \\
\hline Imidazole & 6.440735 & 6.876301 & L-histidine \\
\hline Ascorbic acid & 5.901574 & 6.193334 & Ascorbic acid \\
\hline Nicotinic acid & 4.350079 & 4.326827 & Nicotinic acid \\
\hline BHA & 3.173866 & 3.715493 & BHA \\
\hline TBHQ & 3.137027 & 3.695464 & TBHQ \\
\hline Trolox & 2.767662 & 3.457373 & Trolox \\
\hline BHT & 2.173667 & 3.051788 & Quercetin \\
\hline Quercetin & 2.165725 & 2.375254 & BHT \\
\hline$\beta$-Carotene & 0.544228 & 0.639347 & $\beta$-Carotene \\
\hline$\alpha$-Tocopherol & 0.223702 & 0.238845 & $\alpha$-Tocopherol \\
\hline DABCO & 0.007315 & 0.008435 & DABCO \\
\hline
\end{tabular}

** Arranged from highest to lowest 
Table 3. Comparative evaluation of all the molecules under study for their oscillator strength in both the systems (vacuum versus aqueous system) in their first singlet state of their excited electronic absorption spectrum

\begin{tabular}{|l|l|l|l|l|l|}
\hline \multicolumn{2}{|c|}{ Osc. Str.(vacuum) ** } & \multicolumn{2}{c|}{ Osc. Str.(water) ** } & \multicolumn{2}{l|}{ H-Bond acceptor * } \\
\hline$\beta$-Carotene & 3.71688 & 3.71661 & $\beta$-Carotene & 0 & $\beta$-Carotene \\
\hline Imidazole & 0.179235 & 0.170434 & Imidazole & 1 & Imidazole \\
\hline TBHQ & 0.042443 & 0.043367 & TBHQ & 1 & BHT \\
\hline BHA & 0.040639 & 0.041312 & BHA & 2 & DABCO \\
\hline DABCO & 0.009558 & 0.009558 & DABCO & 2 & $\alpha$-Tocopherol \\
\hline BHT & 0.008328 & 0.008191 & BHT & 2 & BHA \\
\hline$\alpha$-Tocopherol & 0.00751 & 0.007506 & $\alpha$-Tocopherol & 2 & TBHQ \\
\hline Trolox & 0.001709 & 0.001844 & Trolox & 3 & Nicotinic acid \\
\hline L-histidine & 0.000963 & 0.00106 & L-histidine & 4 & L-histidine \\
\hline Rose Bengal & 0.000745 & 0.000492 & Nicotinic acid & 4 & Trolox \\
\hline Ascorbic acid & 0.000538 & 0.000473 & Ascorbic acid & 5 & Rose Bengal \\
\hline Nicotinic acid & 0.000493 & 0.000256 & Rose Bengal & 6 & Ascorbic acid \\
\hline Quercetin & 0.000021 & 0.000012 & Quercetin & 7 & Quercetin \\
\hline
\end{tabular}

*Arranged from lowest to highest ** Arranged from highest to lowest

Table 4. Values of $\log \mathrm{P}$ (octanol/water partition coefficient; modified version XlogP3) obtained from PubChem along with $\mathrm{H}$ bond donor as well as $\mathrm{H}$-bond acceptor have been listed which partially hints about quenching potential of molecules

\begin{tabular}{|l|l|l|l|}
\hline & XlogP3 $* *$ & H-Bond donor & H-bond acceptor \\
\hline B-Carotene & 13.5 & 0 & 0 \\
\hline$\alpha$-Tocopherol & 10.7 & 1 & 2 \\
\hline Rose Bengal & 7.6 & 2 & 5 \\
\hline BHT & 5.3 & 1 & 1 \\
\hline BHA & 3.2 & 1 & 2 \\
\hline Trolox & 2.8 & 2 & 4 \\
\hline TBHQ & 2.8 & 2 & 2 \\
\hline Quercetin & 1.5 & 5 & 7 \\
\hline Nicotinic acid & 0.4 & 1 & 3 \\
\hline Imidazole & -0.1 & 1 & 1 \\
\hline DABCO & -0.2 & 0 & 2 \\
\hline Ascorbic acid & -1.6 & 4 & 6 \\
\hline L-histidine & -3.2 & 3 & 4 \\
\hline
\end{tabular}

** Arranged from highest to lowest 


\section{Acknowledgments}

We are grateful to Council of Scientific and Industrial Research (CSIR), New Delhi for financial support (BSC0121) at CSIR-CIMAP, Lucknow.

\section{Abbreviations}

Photodynamic therapy: PDT

Hydrogen Peroxide/Sodium hypochlorite:

$\mathrm{H}_{2} \mathrm{O}_{2} / \mathrm{NaOCl}$

Lowest Unoccupied Molecular Orbital: LUMO

Singlet oxygen: ${ }^{1} \mathrm{O}_{2}$ or ${ }^{1} \Delta g$

1,4 Diazabicyclo[2.2.2]octane : DABCO

$p$-Nitroso dimethylaniline : RNO

Low density lipoprotein: LDL

Triplet oxygen: ${ }^{3} \mathrm{O}_{2}$ or $3 \sum_{\mathrm{g}}$

Octanol/water partition : $\log \mathrm{P}$ or $\mathrm{X} \log \mathrm{P} 3$

Configuration Interaction: $\mathrm{CI}$

Self-Consistent Reaction Field: SCRF

Self-consistent Field: SCF

N,N-dimethyl-4-nitrosoaniline: RNO,

Dimethyl sulfoxide: DMSO

Butylated hydroxyanisole: BHA

Butylated hydroxytoluene: BHT

Tertbutyl hydroquinone: TBHQ

Di-sodium hydrogen phosphate: $\mathrm{Na}_{2} \mathrm{HPO}_{4}$

Sodium dihydrogen phosphate: $\mathrm{NaH}_{2} \mathrm{PO}_{4}$

Zerner's Spectroscopic parameterization of the Intermediate Neglect of Differential Overlap INDO1/s Hamiltonian: ZINDO

\section{Competing interests}

The authors declare that they have no competing interests.

\section{Open Access}

This article is distributed under the terms of the Creative Commons Attribution License (CC-BY 4.0) which permits any use, distribution, and reproduction in any medium, provided the original author(s) and the source are credited.

\section{References}

Ameta, S.C., Punjabi, P.B., Chobisa, C.S., Mangal, N. and Bhardwaj, R. (1990). Singlet molecular oxygen. Asian Journal of Chemistry Review, 1(2):106-124.

Anouar, el H., Osman, C.P., Weber, J.F., Ismail, N.H.( 2014). UV/Visible spectra of a series of natural and synthesized anthraquinones: experimental and quantum chemical approaches. Springerplus, 3:233.

ArgusLab 4.0, Thompson MA. Planaria Software LLC; Seattle, WA (http://www.arguslab.com)

Bachowski, G. J., Morehouse, K. I. M. M., \& Girotti, A. W. (1988). PorphyrinSensitized photoreactions in the presence of Ascorbate: Oxidation of cell Membrane lipids and hydroxyl radical traps. Photochemistry and Photobiology, 47(5), 635-645.

Bachowski, G. J., Pintar, T. J., \& Girotti, A. W. (1991). Photosensitized lipid peroxidation and enzyme inactivation by membrane-bound Merocyanine 540 reaction mechanisms in the absence and presence of ascorbate*. Photochemistry and Photobiology, 53(4), 481-491.

Buettner, G. R., Kelley, E. E., \& Burns, C. P. (1993). Membrane Lipid-Free Radicals Produced from L1210 Murine Leukemia Cells by Photofrin Photosensitization: An Electron Paramagnetic Resonance Spin Trapping Study. Cancer Research, 53(16), 3670-3673.

Christensen, H. A. F. and R. L. (2008). Excited Electronic States, Photochemistry and Photophysics of Carotenoids. In P. D. H. P. Dr. George Britton, Professor Dr. Dr. h.c Synnøve Liaaen-Jensen (Ed.), Carotenoids Volume 4: Natural Functions (Birkhäuser., pp. 167-188). Boston: Birkhäuser Basel.

Deby-Dupont, G., Deby, C., Mouithys-Mickalad, A., Hoebeke, M., MathyHartert, M., Jadoul, L., Vandenberghe, A. and Lamy, M. (1998). The antibiotic ceftazidime is a singlet oxygen quencher as demonstrated by ultra-weak chemiluminescence and by inhibition of AAP consumption. Biochim Biophys Acta. 1379(1):61-8

DeRosa, M. C., \& Crutchley, R. J. (2002). Photosensitized singlet oxygen and its applications. Coordination Chemistry Reviews, 233-234, 351-371.

Ghazy, R., Azim, S. ., Shaheen, M., \& El-Mekawey, F. (2004). Experimental studies on the determination of the dipole moments of some different laser dyes. Spectrochimica Acta Part A: Molecular and Biomolecular Spectroscopy, 60(1-2), 187-191.

Girotti, A. W., Thomas, J. P., \& Jordan, J. E. (1985). Prooxidant and antioxidant effects of ascorbate on photosensitized peroxidation of lipids in erythrocyte membranes. Photochemistry and Photobiology, 41(3), 267-276.

Greer, A. (2006). Christopher Foote's discovery of the role of singlet oxygen [1O2 (1Delta $\mathrm{g})$ ] in photosensitized oxidation reactions. Accounts of Chemical Research, 39(11), 797-804.

Hooper, M. J., Ankley, G. T., Cristol, D. A., Maryoung, L. A., Noyes, P. D., \& Pinkerton, K. E. (2013). Interactions between chemical and climate stressors: a 
role for mechanistic toxicology in assessing climate change risks. Environmental Toxicology and Chemistry / Setac, 32(1), 32-48.

Jacobsen, J., \& Knak Jensen, S. J. (2007). A mechanism for production of singlet oxygen by acidification of hypochlorite. Chemical Physics Letters, 449(1-3), 135137.

Jakus, J., \& Farkas, O. (2005). Photosensitizers and antioxidants: a way to new drugs? Photochem. Photobiol. Sci., 4(9), 694 698.

Kaliya, O. L., Lukyanets, E. A., \& Vorozhtsov, G. N. (1999). Catalysis and Photocatalysis by Phthalocyanines for Technology, Ecology and Medicine. Journal of Porphyrins and Phthalocyanines, 03(07), 592-610.

Karelson, M. M., \& Zerner, M. C. (1992). Theoretical treatment of solvent effects on electronic spectroscopy. The Journal of Physical Chemistry, 96(17), 69496957.

Kelley, E. E., Domann, F. E., Buettner, G. R., Oberley, L. W., \& Burns, C. P. (1997). Increased efficacy of in vitro Photofrin ${ }^{\circledR}$ photosensitization of human oral squamous cell carcinoma by iron and ascorbate. Journal of Photochemistry and Photobiology B: Biology, 40(3), 273-277.

Koichi, S., \& John, F. K.(2011). Reactive oxygen species in cardiovascular diseases. Free Radic Biol Med, 51(5), 978-992.

Kraljić, I., \& Mohsni, S. El. (1978). A new method for the detection of singlet oxygen in aqueous solutions. Photochemistry and Photobiology, 28(4-5), 577-581.

Melnikova, V. O., Bezdetnaya, L. N., Brault, D., Potapenko, A. Y., \& Guillemin, F. (2000). Enhancement of meta-tetrahydroxyphenylchlorin-sensitized photodynamic treatment on human tumor xenografts using a water-soluble vitamin $E$ analog, Trolox. International Journal of Cancer, 88(5), 798-803.

Melnikova, V., Bezdetnaya, L., Belitchenko, I., Potapenko, A., Merlin, J. L., \& Guillemin, F. (1999). Meta-tetra(hydroxyphenyl)chlorin-sensitized photodynamic damage of cultured tumor and normal cells in the presence of high concentrations of alpha-tocopherol. Cancer Letters, 139(1), 89-95.

Pajouhesh, H., \& Lenz, G. R. (2005). Medicinal Chemical Properties of Successful Central Nervous System Drugs. NeuroRx, 2(4), 541-553.

Rosen, J. E. (1997). Proposed mechanism for the photodynamic generation of 8 oxo-7,8-dihydro-2'-deoxyguanosine produced in cultured cells by exposure to lomefloxacin. Mutation Research/Fundamental and Molecular Mechanisms of Mutagenesis, 381(1), 117-129.

Rosenthal, I., \& Ben-Hur, E. (1992). Ascorbate-assisted, Phthalocyanine-sensitized Photohaemolysis of Human Erythrocytes. International Journal of Radiation Biology, 62(4), 481-486.

Semiempirical Molecular Orbital Methods Michael C. Zerner in Reviews in Computational Chemistry II Ed. Kennith B. Libkowitz and Donald B. Boyd VCS Publishers Inc. 1991, Cpt. 8 p 313-366.

Shevchuk, I., Chekulayev, V., \& Chekulayeva, L. (1998). Enhancement of the efficiency of photodynamic therapy of tumors by t-butyl-4-hydroxyanisole. Journal of Photochemistry and Photobiology B: Biology, 45(2-3), 136-143.

Shevchuk, I., Chekulayev, V., \& Chekulayeva, L. (1998). Enhancement of the efficiency of photodynamic therapy of tumors by t-butyl-4-hydroxyanisole. Journal of Photochemistry and Photobiology B: Biology, 45(2-3), 136-143.

Stockert, J. C., \& Herkovits, J. (2003). Photodynamic toxicity and its prevention by antioxidative agents in Bufo arenarum embryos. Toxicology, 192(2-3), 211218 .

Suppan, P. (1990). Invited review solvatochromic shifts: The influence of the medium on the energy of electronic states. Journal of Photochemistry and Photobiology A: Chemistry, 50(3), 293-330.

Synnove Liaane-Jensen, B. F. L. (2008). Structure and Properties of Carotenoid Cations. In P. D. H. P. Dr. George Britton, Professor Dr. Dr. h.c Synnøve LiaaenJensen (Ed.), Carotenoids Volume 4: Natural Functions (Birkhäuser., pp. 155166). Boston: Birkhäuser Basel.
Thomas, P. A., Devasagayam \& Jayashree, P. Kamat. (2002). Biological significance of singlet oxygen. Indian Journal of Experimental Biology, 40, 680-692

Wilkinson, F., Helman, W. P., \& Ross, A. B. (1995). Rate Constants for the Decay and Reactions of the Lowest Electronically Excited Singlet State of Molecular Oxygen in Solution. An Expanded and Revised Compilation. Journal of Physical and Chemical Reference Data, 24(2).

Zerner, M. C., Loew, G. H., Kirchner, R. F., \& Mueller-Westerhoff, U. T. (1980). An intermediate neglect of differential overlaps technique for spectroscopy of transition-metal complexes. Ferrocene. Journal of the American Chemical Society, 102(2), 589-599.

\section{Cite this article as:}

Fatima, K., Masood, N., \& Luqman, S. (2016). Quenching of singlet oxygen by natural and synthetic antioxidants and assessment of electronic UV/Visible absorption spectra for alleviating or enhancing the efficacy of photodynamic therapy. Biomedical Research And Therapy, 3(2): 514-527. 